\title{
Hearing within Rigid Constrains: Speech Detection while Wearing a Football Helmet
}

\author{
Michael S. Gordon*, Charles H. Brown and Mary Kolbusz
}

University of South Alabama, 307 University Blvd. Mobile, AL 36688-0002, USA

\begin{abstract}
Hearing is a function of the acoustic signal and the properties of the environment that structure the sound. To better understand how hearing is affected by structural properties, the current research investigated the impact of football helmets for hearing. Speech signals were broadcast at three angles of incidence $\left(0^{\circ}, 45^{\circ}\right.$, or $\left.90^{\circ}\right)$ to an artificial dummyhead recording device wearing a football helmet (experimental condition) or without the helmet (control condition) to make binaural recordings using condenser microphones positioned at the location of the tympanic membrane. Acoustic analyses revealed that the helmet caused frequency-specific changes to an auditory signal, including both positive and negative interference that varied in relationship to the angle of incidence. Behavioral studies showed that while; in general, the football helmet made hearing speech more difficult, the magnitude of the decrement was a function of the amount of spectral change induced in the speech signal. Results are discussed in terms of the implications of an ecological approach to hearing and the design of helmets.
\end{abstract}

Keywords: Psychacoustics, hearing, helmets, speech, scene analysis, football.

\section{INTRODUCTION}

Acoustic communication is a function of the acoustic signals and how those signals are propagated through the environment. The combined action of acoustic scatter, reverberation, amplitude fluctuation, frequency-specific attenuation, and so forth distort the signal incident at the listener's ear [1-4]. Hearing then is a function of the acoustic composition of the emitted signal and its subsequent perturbation by the environment. An interesting question is what happens to communication, or specifically speech detection, when a person wears a helmet.

American football is a violent, high-velocity sport. At full-throttle members of opposing teams may collide at velocities exceeding 30 miles per hour. As per its design, the helmet increases the safety of the wearer in the event of a collision. To diminish the risk of head injury, football helmets are composed of a rigid exterior cover overlying a compressible shock-absorbing inner layer. The thick walls and dense exterior of the helmet increase the effective acoustic radius of the skull. The size and shape of the helmet will cause variations in the resonant qualities of the regions around the pinnae, and likely alter the intensity levels, reverberation times, and spectral content of the signal relative to normal listening conditions. In covering the whole of the head, helmets also tend to change the pathways that allow for the passage of acoustic energy to the ears (relative to normal listening conditions), and helmets of different design may partially or completely occlude the opening to the ear canals. For a football player listening to a snap count, or the blocking assignments that precede play, helmet

*Address correspondence to this author at the University of South Alabama, 307 University Blvd. N, Mobile, AL 36688-0002, USA; Tel: 251 460 6546; Fax: 251 460-6320; E-mail: mgordon@jaguar1.usouthal.edu acoustics may amplify errors in speech detection, and the intense level of crowd-noise in some stadium environments may complicate this even further.

The following research investigated how wearing a football helmet altered the detection of high- and lowcontext sentences presented from $0^{\circ}, 45^{\circ}$, and $90^{\circ}$ in noisy backgrounds. We chose specifically to study football helmets because of the extensive covering and the level of protection they offer a wearer. To determine the impact of a football helmet on hearing we performed two kinds of measurements: (1) acoustic measurements with an artificial head and (2) perceptual measurements with young adult listeners. To this end a programmatic series of analyses were made of 12 talker babble (Cosmos Distributing, 2005) [5] to determine precisely how these signals interacted with the helmet relative to normal hearing conditions. Following the acoustic analysis of these recordings, we conducted behavioral measurements to determine the decrements in speech detection for a listener as a function of signal azimuth $\left(0^{\circ}\right.$, $45^{\circ}, 90^{\circ}$ ) while wearing or not wearing a football helmet.

There has been very little previous research investigating how hearing is affected by helmets of any type, and there have been no specific published studies into the acoustics of football helmets per se. Research addressing the acoustics of motorcycle helmets has focused on the tremendous amount of noise induced by wind turbulence [6,7]. In this research the acoustics of helmet design was not specifically tested, and under high turbulent conditions the influence of the helmet on hearing was found to be quite minimal. Military researchers have also considered the impact of helmets on communication [8-10]. In general this research has examined in-helmet electronic communication systems rather than analyses of how the helmet affects the perception of signals in the environment that are external to the electronic 
communication link, or how the helmet affects face-to-face communication. One relevant finding from the military helmet research is that the amount of ear obstruction of a helmet may be the most predictive design element in terms of communication [11]. In the current study we also consider how the ear hole design parameters of football helmets might relate to communication.

\section{EXPERIMENT 1: ACOUSTIC MEASUREMENTS OF 12-TALKER BABBLE WITHIN A FOOTBALL HELMET}

This initial analysis was designed to measure how a football helmet affects the acoustic transmission of a signal to a listener. In the following measurements 12-talker babble was recorded from a head either wearing or not wearing a football helmet. These recordings were analyzed to provide some description of how a helmet can affect an acoustic signal.

\section{Materials and Methods}

A dummyhead simulation of an adult male human head (Neumann KU-100) was positioned on a tripod in a doublewalled sound isolation booth. Audio stimuli (12-talker babble samples, Cosmos Distributing, 2005) were broadcast via a Bowers \& Wilkins DM601 S3 loudspeaker powered by a Kenwood 107VR Audiovisual Receiver. The loudspeaker was located $0.76 \mathrm{~m}$ from the head and the broadcast level (measured at the center of the head) was 95 dBA. The Neumann KU-100 contains a binaural recording system with condenser microphones embedded in the ear canals at the position of the tympanic membrane. Binaural audio recordings were created with and without the football helmet at orientations of $0^{\circ}, 45^{\circ}$, and $90^{\circ}$. The different broadcast azimuths were achieved by rotating the head relative to the position of the microphone. The football helmet was an ADAMS Pro-Elite (X-large) helmet with Pro-50 chinstrap, and facemask. The helmet was fit snugly over the artificial head with the helmet's earholes aligned over the ear canals of the dummy (see Fig. 1). The output of the Neumann KU-100 binaural microphone system was fed directly into an Alienware VTK-76 custom computer and digitized at $48 \mathrm{kHz}$ using Adobe Audition 1.5 software.

To compare the changes in the signal as caused by the helmet, a Blackmann-Harris Fast Fourier Transform (FFT) was completed on each of these samples to determine the changes in power across the normal hearing spectrum, in 86 $\mathrm{Hz}$ intervals (e.g., 0-86 Hz, 87-172 Hz, 173-259 Hz, $\sim 19,983-20,069 \mathrm{~Hz}$ ). Computation of each of these 234 spectral bands was computed for 15 babble samples taken with the helmet and 15 matched samples (i.e., the same babble recording) taken without the helmet. After generating these FFTs, the difference in dBs was calculated between the helmet and no-helmet samples, and averaged across the 15 matched pairs. The difference scores for the matched pairs of stimuli were calculated to simplify the comparison of the magnitude of power variations caused by the helmet, across the three listening angles.

\section{RESULTS AND DISCUSSION}

Fig. (2) shows the average difference scores computed across the tested frequency range for each of the three listening angles tested. Summaries of each of these three listening angles demonstrate the peak resonances and attenuated frequencies as caused by the helmet.

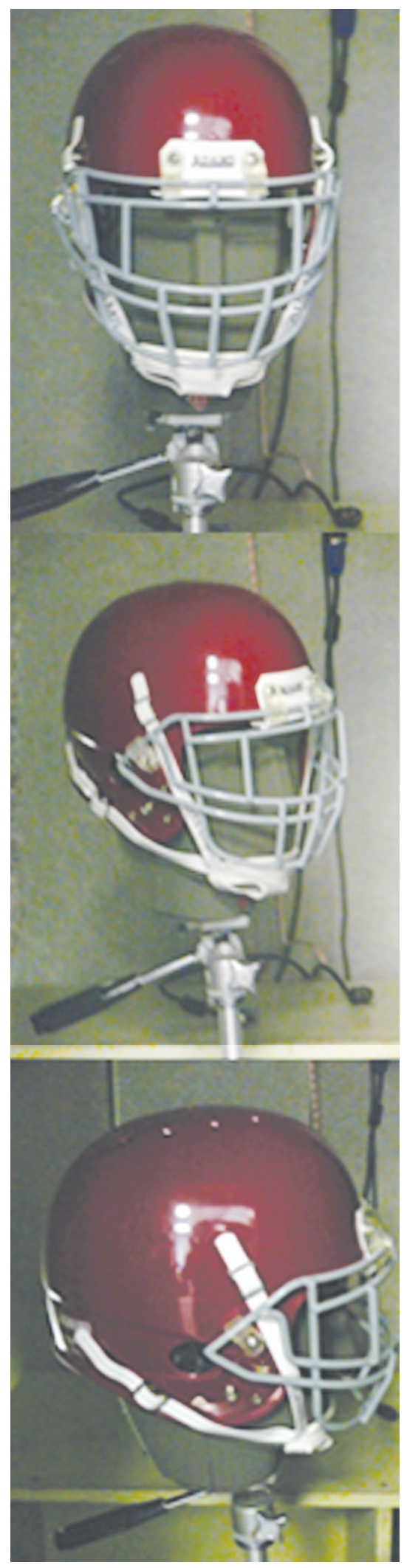

Fig. (1). Views of the dummyhead wearing the helmet as used for generation of the recordings from $0^{\circ}$ (top), $45^{\circ}$ (middle), and $90^{\circ}$ (bottom), respectively. 


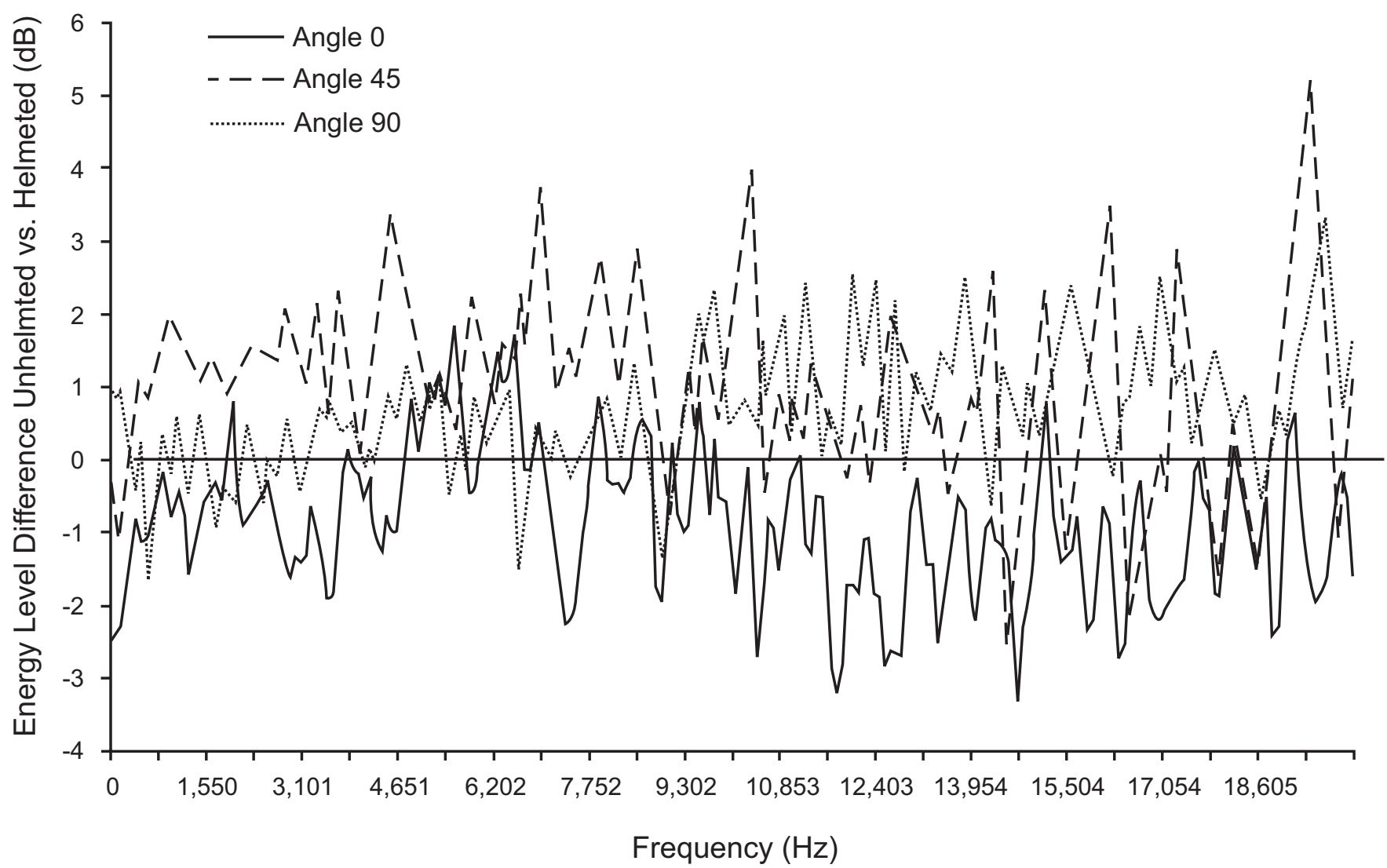

Fig. (2). Differences between the FFT power analysis for recordings taken with the football helmet relative to those with no helmet. For comparison purposes across the listening angles, all power levels are reported as the average dB difference between the helmet/no-helmet conditions. Higher values indicate potential resonances and constructive interference from the helmet and lower values indicate helmetproduced attenuation and destructive interference.

Helmet vs No-Helmet at $0^{\circ}$ : On average the helmet caused a loss of $-0.86 \mathrm{~dB}$ across the audible spectral range, with a standard deviation of $0.98 \mathrm{~dB}$ for the $0^{\circ}$ listening angle. Given the open front style of the football helmet, and the limited amount of ear covering at this angle, it may not be surprising that the overall differences between helmet and non-helmet listening conditions were fairly small. As shown in Fig. (2), the greatest attenuation was found between $11,000-15,000 \mathrm{~Hz}$, with its nadir at $14,800 \mathrm{~Hz}(\mathrm{a}-3.3 \mathrm{~dB}$ loss). While several other areas with energy loss can be highlighted in the higher frequency spectral bands, little power loss occurred for frequencies less than $6,000 \mathrm{~Hz}$, amidst the central parts of the spectral range associated with speech (i.e., about $250-6,000 \mathrm{~Hz}$ ). In the lower range, there was moderate diminishment of power at $180 \mathrm{~Hz}(-1.8 \mathrm{~dB}$ of attenuation) and between 2,600-3,200 $\mathrm{Hz}$ there was an approximately $-1.2 \mathrm{~dB}$ power loss. The helmet produced a $1.9 \mathrm{~dB}$ increase in signal level between 4 and $6 \mathrm{kHz}$ relative to the no-helmet condition. Additional increments of about 1 $\mathrm{dB}$ were observed near $7,850 \mathrm{~Hz}$ and $15 \mathrm{kHz}$.

Given the small and relatively constant power shift within the central part of the frequency range associated with most speech signals, only a small affect on speech detection performance would be expected for listeners wearing a helmet at this angle. One unexpected finding was the observation of an increase in intensity for some portions of the spectrum when the helmet was fitted on the artificial head relative to the un-helmeted condition. When the helmet is positioned on the head the sound wave can reach the ear canal through at least two distinct paths. One path is provided by the ear holes in the side of the helmet which were aligned with the ear canal. A second path is provided by the space between the side of the face and the interior of the helmet relative to the cheek and ear canal. Two or more pathways provide for the opportunity for constructive and destructive interference yielding both amplitude gains and losses, as displayed in Fig. (2). Frequency-specific resonances in the helmet may also contribute to the observation of power gains and losses, in comparison to the helmeted and un-helmeted conditions. More material science research is needed to evaluate the structural elements that contribute to constructive and destructive interference. It is possible that the constructive interference effect may account for anecdotal reports that some players feel that the helmet acts to improve their hearing. However, players differ with respect to their head and face morphology, and different brands of helmets, different size helmets, and different face masks and chin straps may all play a role in an individual player's auditory experience when fitted with their helmet.

Helmet vs No-Helmet at $45^{\circ}$ : On average the helmet caused an increase in power of $0.93 \mathrm{~dB}$ of attenuation across the audible spectral range, with a standard deviation of 1.21 
$\mathrm{dB}$ for the $45^{\circ}$ listening angle. Presumably the rapid and loud echo that occurred around the ear contributed to this average increase in energy while wearing the helmet at this listening angle. As can be seen in Fig. (2), between $600-3,500 \mathrm{~Hz}$ the signal gained an average $1.5 \mathrm{~dB}$ in energy. Peak energy gains were found at $4,500 \mathrm{~Hz}, 7,000 \mathrm{~Hz}, 10,250 \mathrm{~Hz}$, and $16 \mathrm{kHz}$ with an average spike of $3 \mathrm{~dB}$, and at $19.5 \mathrm{kHz}$ with a gain of over $5 \mathrm{~dB}$.

There were also spectral regions that displayed large levels of power loss at this listening angle. Fig. (2) shows the largest power losses at 14.5 and $16.5 \mathrm{kHz}$, at nearly $-2.5 \mathrm{~dB}$. It is notable that in the speech range, between $250-6,000 \mathrm{~Hz}$ the energy level in the helmet was higher than in the nohelmet condition for the entire spectrum. While constructive interference produces an amplitude gain, it may also be associated with phase shifts in portions of the audio spectrum, and intensity increments in background noise, potentially making signal detection more difficult.

Helmet vs No-Helmet at $90^{\circ}$ : On average the helmet caused an amplitude increase of $0.68 \mathrm{~dB}$ across the audible spectral range, with a standard deviation of $0.82 \mathrm{~dB}$ for the $90^{\circ}$ listening angle. The fit of the helmet positioned the ear hole nearly centered over the ear of the dummyhead. Hence, this listening angle may have generated a moderate echo around the ear, increasing the overall levels. Most of these increases in signal level were found in the high-frequency region of the spectrum. Fig. (2) shows the peak areas of constructive interference were all found over $9,000 \mathrm{~Hz}$, with the largest gains from $19-20 \mathrm{kHz}(3 \mathrm{~dB})$, and with spikes at $11,12,13.5$, and $16.2 \mathrm{kHz}$ of approximately $2.5 \mathrm{~dB}$. Fig. (2) also shows the nadir energy levels at 500, 6,500, and 9,000 $\mathrm{Hz}$, each with losses of about $-1.5 \mathrm{~dB}$. While there was amplitude variability in the speech range, in general this variation was less than $\pm 1 \mathrm{~dB}$. Hence, this analysis suggests that similar to the $0^{\circ}$ listening angle, hearing should be relatively unaffected by wearing the helmet and listening at $90^{\circ}$.

In considering these analyses from the three listening angles, it is evident that the $45^{\circ}$ listening angle causes the greatest disturbance to the signal, with a large standard deviation, and the largest overall amplitude shift while wearing the helmet. An interesting question is how these analyses will correspond to listening performance in the following experiments with the root mean square (RMS) power levels equated. By equating the RMS values the mean intensity levels will be the same in each listening condition (helmet, no-helmet, and at each listening angle), however, because of the power shifts to the signal caused by the helmet, there will still be significant distortion for the listeners.

\section{EXPERIMENT 2: PERCEPTUAL MEASUREMENTS WITH AND WITHOUT A HELMET}

\section{Stimulus Generation}

Two types of acoustic stimuli were used to create the auditory recordings for this experiment. Sentences were drawn from the Revised-SPIN lists 7 and 8 [5, 12]. These stimuli were professionally recorded single sentences spoken by a male actor. The 12-talker babble noise files associated with SPIN lists 7 and 8 were also used (Cosmos Distributing, 2005). In addition to analyzing the differences between hearing with and without the helmet, sentences using high and low semantic context were tested. The advantage of manipulating the semantic context was to allow some initial investigation into how changes in signal detection might interact with higher-level cognitive mechanisms. Potentially more difficult listening situations might cause listeners to divert cognitive resources away from semantic processing, thus limiting the contribution of semantic context.

To create analogous recordings with and without a football helmet, these sentences and babble files were all rerecorded using the same apparatus and techniques described for the acoustic measurements.

\section{Materials}

The sentence materials were the Revised SPIN lists 7 and $8[5,12]$ and their associated 12-talker babble-noise files (Cosmos Distributing, 2005). Each sentence list was composed of 50 sentences ( 25 were high-context sentences and 25 were low-context sentences). In the high-context sentences the final word was semantically and syntactically predicted by the sentence, e.g., "the boy kicked the ball"; in the low-context sentences the final word was syntactically, but not semantically predicted by the sentence, e.g., "the boy thought about the ball". The re-recorded sentences described in the Analysis section were used as stimuli. Sentence recordings made without a helmet at $0^{\circ}$ were paired with the babble signals recorded at $0^{\circ}$ without a helmet, and the sentence recordings made with a helmet at $0^{\circ}$ were paired with the babble signals recorded at $0^{\circ}$ with a helmet. To equalize power levels an RMS analysis was completed and all stimuli were set to match the same levels (both signal and babble files).

\section{Perceptual Testing Apparatus}

Sentences were presented to listeners from an Alienware VTK-76 custom computer via a Tucker-Davis Technologies (TDT) System 3 programmable attenuator using a custom visual basic script. The TDT outputted the sound files to a set of Sennheiser HDA 200 headphones. Stimuli were calibrated by playing the sound files over the headphones to the dummyhead and recording the levels on a Sencore SoundPro Audio Integrator SP395.

Perceptual experiments were conducted within a doublewalled sound attenuating chamber that contained an Acer AL 1521 monitor, remotely connected to the Alienware computer.

\section{Procedure}

At the onset of the experiment the participants were instructed that they were to listen to sentences in a noisy background and try to repeat what they had heard. Participants answered a short series of questions regarding their age, hearing ability, and language ability. For the 100 sentences presented, participants listened to the stimulus and 
were asked to repeat the final word. The responses were given verbally by the participant and recorded via the experimenter. The remote monitor in the sound attenuating chamber allowed participants to see and confirm that the recorded responses were correct. After receiving confirmation, the experimenter triggered playback of the next stimulus.

Participants were counterbalanced between the lists, such that half heard the recordings made with the helmet for SPIN list 7 and half received the helmeted recordings from SPIN list 8 , with the other list recorded without the helmet. By using the two lists in this manner it allowed the presentation of an equal balance of the sentence materials between the two helmet conditions. To avoid learning strategies for the hearing within the helmet condition, the two lists were randomized together and presented in a single block. The randomization procedure used in this experiment generated a novel presentation order for each participant.

All sentences were presented binaurally to listeners with background babble at a signal-to-noise $(\mathrm{S} / \mathrm{N})$ ratio of $0 \mathrm{~dB}$ (unless otherwise stated), and signal level at $65 \mathrm{dBA}$ weighted. The experiment lasted approximately 45 minutes per subject. After the final sentence, participants were debriefed about the purpose of the experiment.

\section{Participants}

The participants were individuals, with no reported hearing difficulties, that were recruited from the General Psychology courses at the University of South Alabama. Participants were offered either course credit or monetary reimbursement (\$7/hour) for participation. All participants were native English speakers who had not previously participated in this or any related speech experiment. Only participants who reported having good, uncorrected hearing were included in this research. Fifteen participants were tested with stimuli recorded at the $0^{\circ}$ azimuth condition, 30 were tested at the $45^{\circ}$ condition, and 21 were assigned to the $90^{\circ}$ azimuth condition, yielding a total of 56 participants. In the section that follows we report the results for Experiment $2 \mathrm{a}, 2 \mathrm{~b}$, and $2 \mathrm{c}$ for the $0^{\circ}, 45^{\circ}$ and $90^{\circ}$ conditions, respectively. Additional tests were conducted at $45^{\circ}$ and $90^{\circ}$ at an improved $\mathrm{S} / \mathrm{N}$ ratio $(4 \mathrm{~dB})$. These additional tests (Experiment $2 \mathrm{~d}$ and $2 \mathrm{e}$ ) were conducted because the $0 \mathrm{~dB}$ $\mathrm{S} / \mathrm{N}$ ratio was found to be too difficult at these lateral azimuths. In Experiments $2 \mathrm{~d}$ and $2 \mathrm{e}$ an additional 36 subjects were tested, with 18 subjects assigned to each condition.

\section{Results and Discussion}

\section{Experiment 2a: $0^{\circ}$ Azimuth Condition}

Fig. (3a) shows the responses to high- and low-context sentences for participants in the un-helmeted and helmeted recorded conditions when listening to speech originating from $0^{\circ}$. As can be seen in this figure, in general performance was better in the normal compared to the helmeted listening conditions. Performance also tended to be (a)

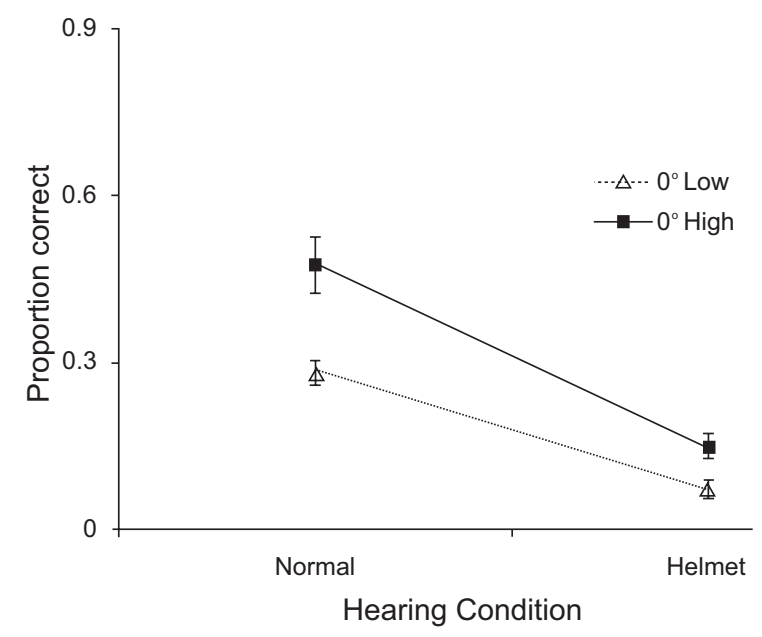

(b)

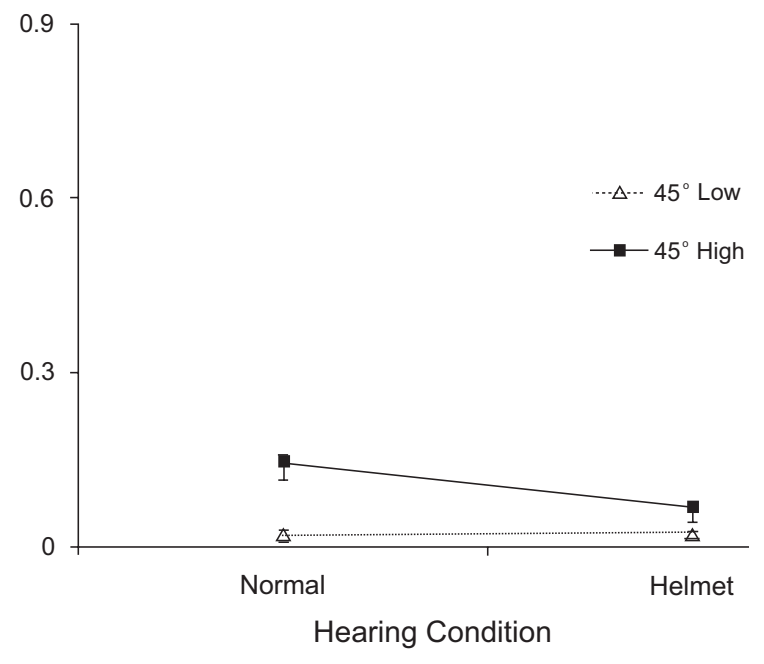

(c)

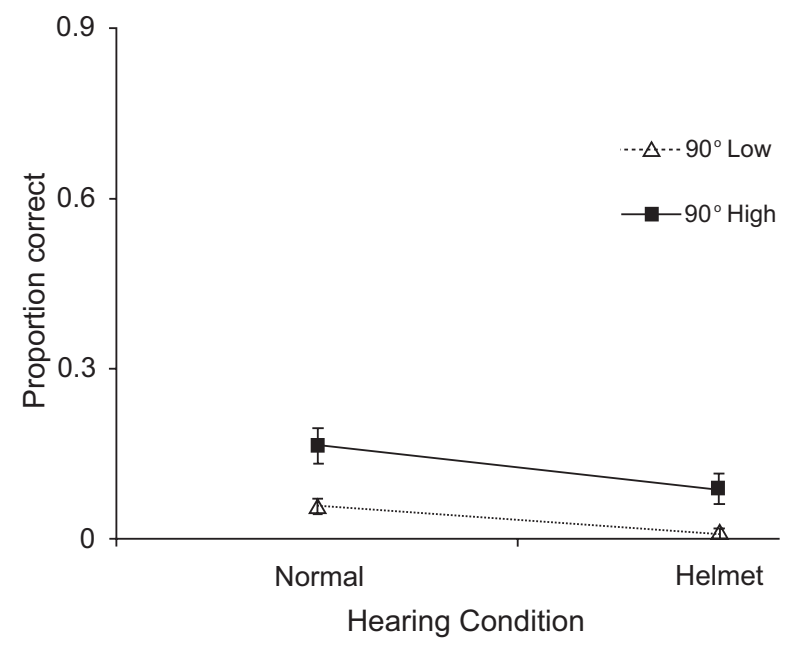

Fig. (3). (a-c) Proportion of correct responses to high and low context sentences originating from $0^{\circ}$ (a), $45^{\circ}$ (b), and $90^{\circ}$ (c). Sentences were recorded normally or under a football helmet with the signal presented at $65 \mathrm{dBA}$-weighted and the $\mathrm{S} / \mathrm{N}$ at $0 \mathrm{~dB}$. 
better with the high-context sentences compared to lowcontext sentences. Statistical analysis confirmed these findings using a within-subjects Context (high $v$ s low) by Helmet (helmet $v s$ normal) by Counterbalance ANOVA with Counterbalance as a between subjects factor.

Results revealed main effects of Helmet $(F[1,14]=$ $90.01, p<.001, \eta \mathrm{p} 2=.87)$, performance was more accurate without a helmet, and Context $(F[1,14]=19.52, p<.005$, $\eta \mathrm{p} 2=.58)$, performance was more accurate with the highcontext sentences. Analyses also revealed an interaction between Helmet and Context $(F[1,14]=8.39, p<.05, \eta p 2=$ .38). The interaction between Helmet and Context (the slopes of the lines differed) suggest that performance differences between the high- and low-context sentences were greater for the normal listening conditions compared to the helmeted listening conditions.

What is evident in the data is that the performance was reduced to very low levels in the helmeted listening conditions. Hence it may be that floor effects within the difficult hearing conditions reduced any potential advantages from the high-context sentences.

\section{Experiment 2b: $45^{\circ}$ Azimuth Condition}

As shown in Fig. (3b) the results revealed main effects of Helmet $(F[1,29]=23.934, p<.0001, \eta p 2=.452)$, performance was more accurate without a helmet, and Context $(F[1,29]=68.9, p<.0001, \eta p 2=.704)$, performance was more accurate with the high-context sentences.

Analyses also revealed an interaction between Helmet and Context $(F[1,29]=45.249, p<.0001, \eta p 2=.609)$. The interaction between Helmet and Context demonstrates that performance differences between the high- and low-context sentences were greater for the normal listening conditions than in the helmeted listening conditions. Statistically these findings paralleled the case for the $0^{\circ}$ azimuth condition. However, the proportion correct was about $1 / 3^{\text {rd }}$ of the value reported for Experiment 2a.

\section{Experiment 2c: $90^{\circ}$ Azimuth Condition}

As displayed in Fig. (3c) the results revealed main effects of Helmet $(F[1,20]=22.887, p<.0001, \eta p 2=.534)$, performance was more accurate without a helmet, and Context $(F[1,20]=13.558, p<.001, \eta p 2=.404)$, performance was more accurate with the high-context sentences. Unlike the case for the $0^{\circ}$ and $45^{\circ}$ azimuth conditions no interaction was found between Helmet and Context $(F[1,20]=1.506, p=.234, \eta \mathrm{p} 2=.07)$. As noted in Experiment $2 \mathrm{~b}$ the main effects of the presence of absence of the helmet, and the high and low word context were significant, but the proportion of correct responding was depressed relative to the $0^{\circ}$ azimuth condition.

\section{Experiment $2 a, 2 b \& 2 c$ Summary}

At lateral azimuths speech reception with a $0 \mathrm{~dB} S / \mathrm{N}$ ration was likely too difficult, and the proportion of correct response was depressed relative to the $0^{\circ}$ azimuth condition. This possibility was explored by repeating Experiment $2 \mathrm{~b}$ and $2 \mathrm{c}$ with an improved $\mathrm{S} / \mathrm{N}$ ratio. Experiment $2 \mathrm{~d}$ and $2 \mathrm{e}$ were identical to Experiment $2 b$ and $2 c$ except that the $S / N$ ratio was improved to $4 \mathrm{~dB}$. Eighteen subjects served in each of the groups.

\section{Experiment 2d: $45^{\circ}$ Azimuth Condition (4 dB S/N)}

Fig. (4a) shows the responses to high- and low-context sentences for participants in the normal and helmeted listening conditions for speech presented from $45^{\circ}$ azimuth. As can be seen in this figure, in general performance was better in the high-context conditions than with low-context sentences. Performance also tended to be better in the normal listening conditions compared to the helmeted condition.

(a)

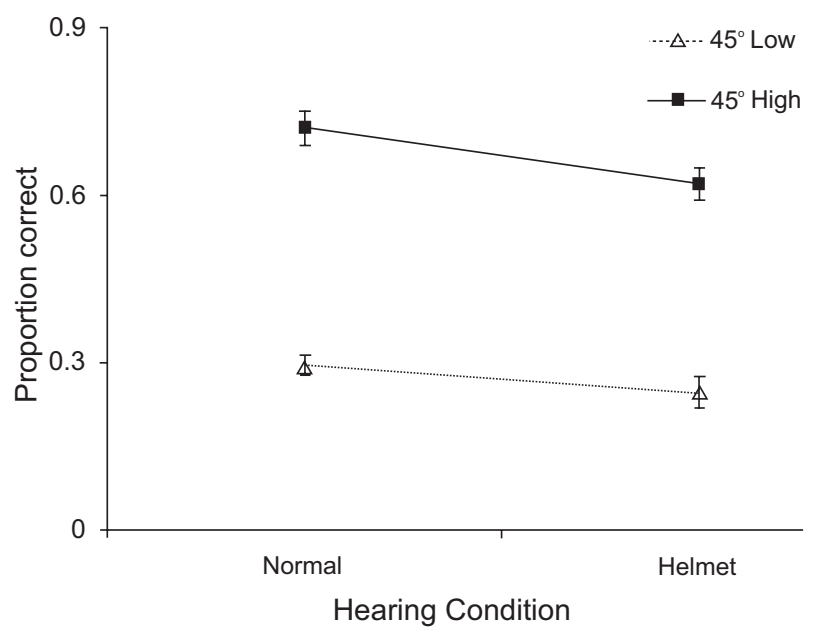

(b)

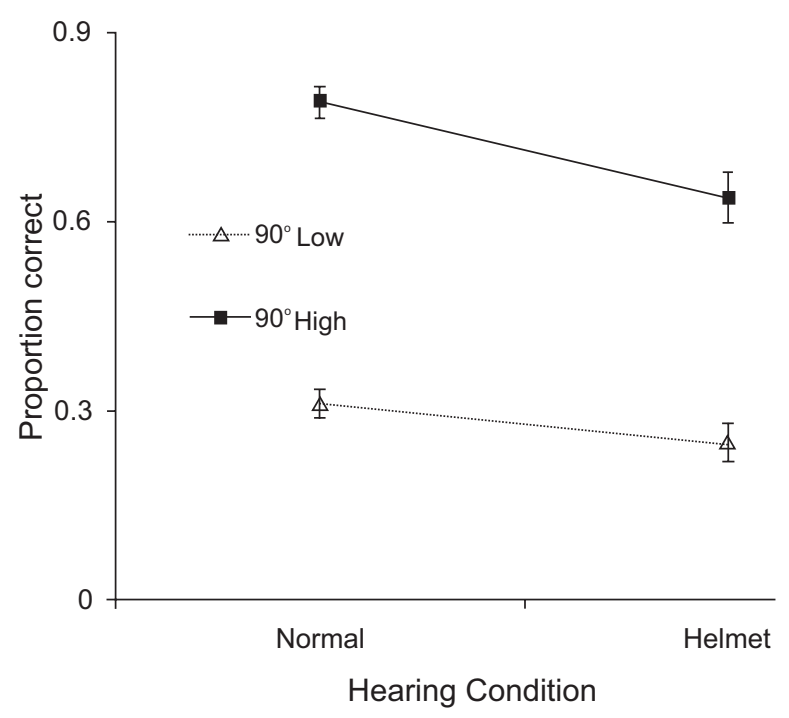

Fig. (4). (a, b) Proportion of correct responses to high and low context sentences originating from $45^{\circ}(\mathbf{a})$, and $90^{\circ}(\mathbf{b})$. Sentences were recorded normally or under a football helmet with the signal presented at $65 \mathrm{dBA}$-weighted and the $\mathrm{S} / \mathrm{N}$ at $4 \mathrm{~dB}$.

Statistical analysis confirmed these findings using a within-subjects Context (high vs low) by Helmet (helmet vs normal) ANOVA with Counterbalance as a between subjects 
factor. Participants performed more accurately in the normal compared to the helmeted listening conditions $(F[1,16]=$ $11.03, p<.005, \eta \mathrm{p} 2=.41$ ). Performance was better for participants with the high-context compared to the lowcontext sentences $(F[1,16]=1.47, p<.001, \eta \mathrm{p} 2=.96)$. Overall, the results of Experiment 2d are similar to that of Experiment $2 b$, with a clear influence of both context and the helmet on performance. The notable differences between Experiments $2 \mathrm{~b}$ and $2 \mathrm{~d}$ is that when the $\mathrm{S} / \mathrm{N}$ ratio was improved to $4 \mathrm{~dB}$ there was no interaction between helmet and context $(F[1,16]=1.07, p>.1, \eta \mathrm{p} 2=.12)$, and there was an overall improvement in performance with this easier $\mathrm{S} / \mathrm{N}$ ratio $(0 \mathrm{~dB}$ vs $4 \mathrm{~dB})$. As opposed to Experiment $2 \mathrm{~b}$, in Experiment $2 \mathrm{~d}$ the helmet simply caused a linear translation of the context effects across the listening conditions and an interaction was not observed. Hence, it is likely that the interaction in $2 b$ (in which the context advantage was larger for participants without the helmet), may have been related to floor levels of performance in some participants listening to the helmeted condition. Hence, subjects were unable to exhibit a performance benefit from the high-context condition.

\section{Experiment 2e: $90^{\circ}$ Azimuth Condition (4 dB S/N)}

Fig. (4b) shows the responses to high- and low-context sentences for participants in the normal and helmet recorded conditions when listening to speech originating at $90^{\circ}$. As can be seen in this figure, in general performance was better in the normal than helmeted conditions. Performance also tended to be better with the high-context sentences than the low-context sentences.

Statistical analysis confirmed these findings using a within-subjects Context (high vs low) by Helmet (helmet $v s$ normal) by Counterbalance ANOVA with Counterbalance as a between subjects factor. There was a main effect of Helmet $(F[1,16]=33.69, p<.001, \eta p 2=.68)$; participants performed better in the normal than helmeted listening conditions. There was also a main effect of Context $(F[1,16]$ $=225.91, p<.001, \eta p 2=.93$ ) demonstrating the better performance by participants with the high- than low-context sentences. There was an interaction between the variables Helmet and Context $(F[1,16]=5.81, p<.05, \eta p 2=.27)$. This interaction is a result of the larger difference between the high- and low-context sentence performances in the Normal listening conditions compared to that with the Helmet. This observation suggests that the absence of an interaction noted in Experiment 2c was due to sampling error when the $\mathrm{S} / \mathrm{N}$ ratio was too low. While this interaction mirrors the results in Experiment 2c, it is interesting to note that in Experiment $2 \mathrm{e}$ the performance tended to stay above the floor levels found in Experiment 1. Hence the results in Experiment 2e may be more consistent with the hypothesis that participants are having some difficulty gaining as large an advantage from the context in the difficult helmeted listening condition than in the normal listening condition.

\section{Experiment 2d and 2e Summary}

The comparison of the results at lateral azimuths $\left(45^{\circ}\right.$ and $90^{\circ}$ ) at $0 \mathrm{~S} / \mathrm{N}$ and $4 \mathrm{~S} / \mathrm{N}$ ratios show that the high and low context effect observed at $0^{\circ}$ azimuth also occurs at lateral azimuths. However, when the $\mathrm{S} / \mathrm{N}$ ratio is too challenging even the high-context words were misperceived. We conclude that high-context cannot compensate for a marginal $\mathrm{S} / \mathrm{N}$ ratio at lateral azimuths.

\section{EXPERIMENT 3: COMPARISONS BETWEEN HEAR- ING AT $0^{\circ}, 45^{\circ}$, AND $90^{\circ}$}

\section{Stimulus Generation}

The stimuli generated using the helmet for Experiment 2 were used in Experiment 3. The helmeted recordings made at $0^{\circ}, 45^{\circ}$, and $90^{\circ}$ were presented.

\section{Perceptual Testing Apparatus \& Materials}

The apparatus and materials were the same as used in Experiment 2.

\section{Procedure}

The procedure was the same as Experiment 2 with the following exceptions. Participants were asked to listen to recordings made at two of the three azimuths (i.e., $0^{\circ} v s 45^{\circ}$, $0^{\circ}$ vs $90^{\circ}, 45^{\circ} v s 90^{\circ}$ ). As in Experiment 2, two lists (and hearing angles) were randomized together and presented in a single block presentation. The randomization procedure used in this experiment generated a novel presentation order for each participant.

All sentences were presented binaurally to listeners with background babble at a signal-to-noise $(\mathrm{S} / \mathrm{N})$ ratio of $0 \mathrm{~dB}$ (unless otherwise stated), and signal level at $65 \mathrm{dBA}$ weighted. The experiment lasted approximately 45 minutes per subject. After the final sentence, participants were debriefed about the purpose of the experiment.

\section{Participants}

The participant recruitment and selection criteria duplicated those utilized for Experiment 2. In Experiment 3a, 21 participants were tested at $0^{\circ}$ and $45^{\circ}$ azimuth. In Experiment $3 \mathrm{~b}, 38$ participants were tested at $0^{\circ}$ and $90^{\circ}$ azimuth. In the final condition, Experiment $3 \mathrm{c}, 18$ participants were tested at $45^{\circ}$ and $90^{\circ}$ azimuth. In total 67 participants were tested across these three conditions.

\section{Experiment 3a: Comparing $0^{\circ}$ and $45^{\circ}$ Hearing Conditions}

As shown in Fig. (5a) subjects performed better at $0^{\circ}$ compared to $45^{\circ}(F[1,19]=5.57, p<.05, \eta p 2=.23)$, and performance was more accurate in the high-context conditions $(F[1,19]=37.90, p<.001, \eta \mathrm{p} 2=.67)$. There was no effect for counterbalancing the order of the stimuli $(p>$ $.05)$ nor was an interaction found between Angle and Context $(p>.05)$. Overall performance in this experiment replicated the findings in Experiments 2a and 2c for hearing with a helmet.

\section{Experiment 3b: Comparing $0^{\circ}$ and $90^{\circ}$ Hearing Conditions}

As shown in Fig. (5b) subjects performed better at $0^{\circ}$ compared to $90(F[1,36]=41.02, p<.001, \eta p 2=.53)$, and performance was better for high-context sentences $(F[1,36]$ $=80.14, p<.001, \eta \mathrm{p} 2=.69)$. These findings matched those 
(a)

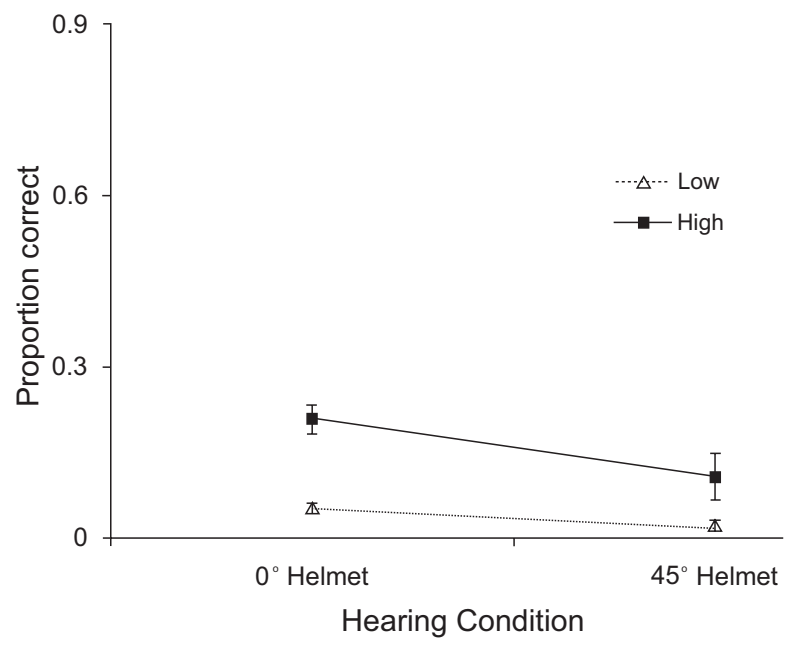

(b)

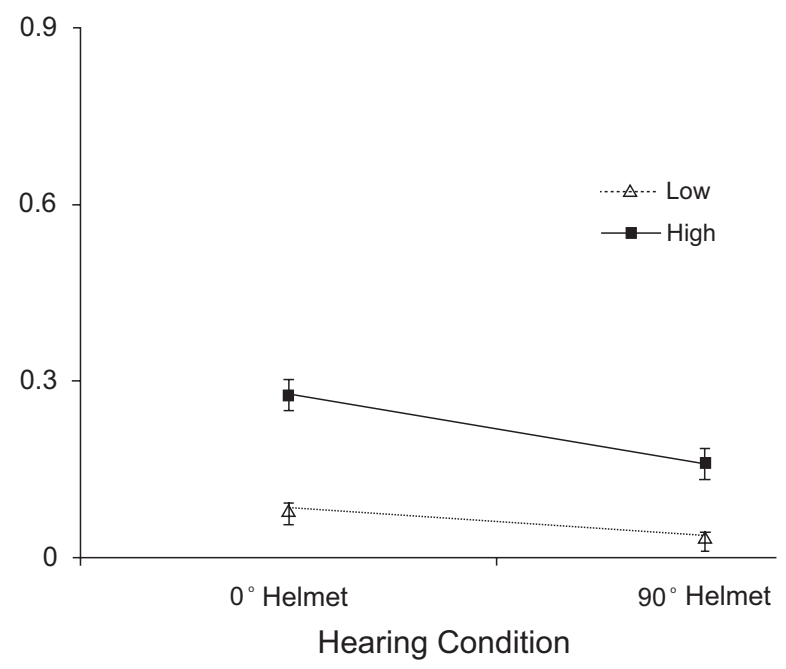

(c)

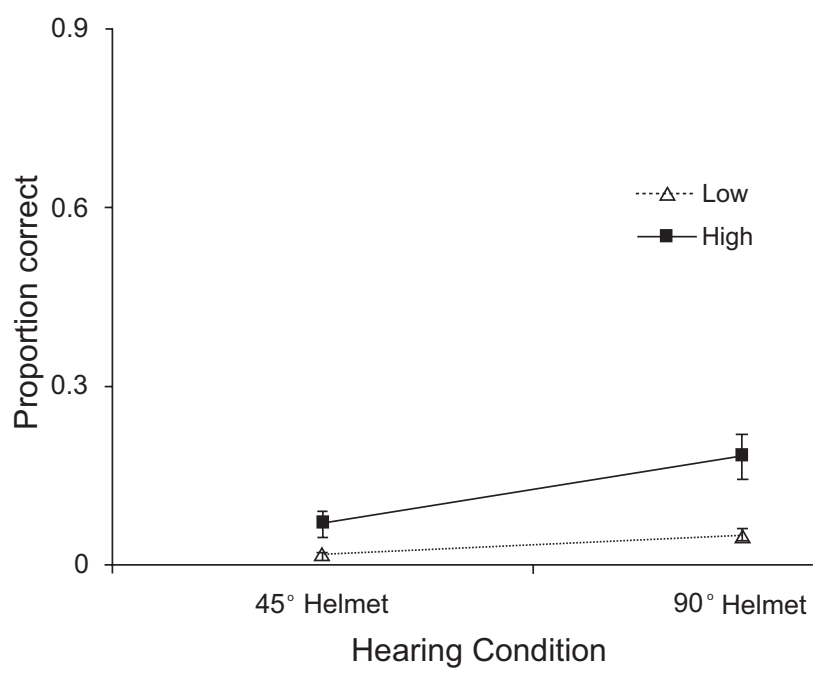

(Fig. 5) contd.....

(d)

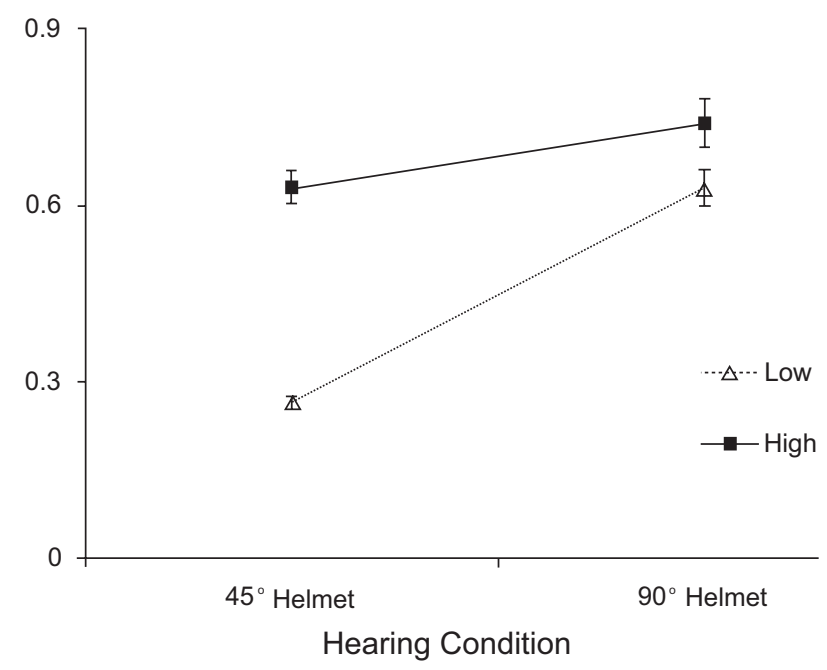

Fig. (5). (a-d). Proportion of correct responses to high and low context sentences originating from $0^{\circ}$ vs $45^{\circ}$ (top-left), $0^{\circ} v s 90^{\circ}$ (top-right), $45^{\circ}$ vs $90^{\circ}$ low S/N (bottom-left), and $45^{\circ}$ vs $90^{\circ}$ high $\mathrm{S} / \mathrm{N}$ (bottom-right). Sentences were recorded with a football helmet with the signal presented at $65 \mathrm{dBA}$-weighted and the $\mathrm{S} / \mathrm{N}$ at $0 \mathrm{~dB}$, except for (d) which was presented at $\mathrm{S} / \mathrm{N} 4 \mathrm{~dB}$.

for Experiment 3a, however, an interaction between angle and context was also observed $(F[1,36]=7.19, p<.05, \eta p 2$ $=.17)$. The interaction suggests that there was a larger advantage using the high context sentences at from $0^{\circ}$ compared to that for the $90^{\circ}$ azimuth condition. There was also a main effect of counterbalance $(F[1,36]=12.88, p$ $<.05, \eta p 2=.26)$ and a three-way interaction between counterbalance, angle, and context was observed $(F[1,36]=$ $49.71, p<.001, \eta \mathrm{p} 2=.58)$. This interaction may suggest that with SPIN List 7 there was a larger context affect for $0^{\circ}$ than $90^{\circ}$, but that this difference was not found for SPIN List 8.

\section{Experiment 3c: Comparing $45^{\circ}$ and $90^{\circ}$ Hearing Conditions}

As can be seen in Fig. (5c) subjects performed better at $90^{\circ}$ compared to that at $45^{\circ}$ azimuth $(F[1,17]=13.94, p<$ $.005, \eta \mathrm{p} 2=.45)$, and subjects performed better with highcontext sentences $(F[1,17]=17.42, p<.001, \eta p 2=.51)$. A significant interaction between Angle and Context $(F[1,17]=$ $6.00, p<.05, \eta \mathrm{p} 2=.26$ ) was also observed. This finding indicates that the difference in performance between highcontext and low-context sentences was greater at $45^{\circ}$ compared to that at $90^{\circ}$ azimuth. There was no main effect of Counterbalance $(p>.05)$ in this comparison.

\section{Experiment 3d: Comparing $45^{\circ}$ and $90^{\circ}$ Hearing Conditions with $S / N$ of 4 dB}

Experiment 3d replicates Experiment $3 \mathrm{c}$ at a more favorable $\mathrm{S} / \mathrm{N}(4 \mathrm{~dB})$, and the results of the two experimental conditions were very similar. As can be seen in Fig. (5d) participants performed better at $90^{\circ}$ compared to that at $45^{\circ}$ 
azimuth $(F[1,13]=95.98, p<.001, \eta \mathrm{p} 2=.88)$, and subjects performed better with high-context sentences $(F[1,13]=$ 137.66, $p<.001, \eta \mathrm{p} 2=.91)$. A significant interaction between Angle and Context was observed $(F[1,13]=23.07$, $p<.001, \eta \mathrm{p} 2=.64)$. The difference in performance between high-context and low-context sentences was greater at $45^{\circ}$ compared to that $90^{\circ}$ azimuth. There was no main effect of Counterbalance $(p>.05)$ in this comparison.

\section{GENERAL DISCUSSION AND CONCLUSIONS}

The helmet influenced the accuracy of speech reception in several ways. Our acoustic analysis demonstrated the presence of constructive and destructive interference caused by the helmet at the three listening angles of incidence. At $0^{\circ}$ and $90^{\circ}$ constructive and destructive interference patterns tended to occur outside of the speech range, while at $45^{\circ}$ a power increase in the signal produced by constructive interference was observed within the speech range. The results from the acoustical measurements suggest that speech accuracy would change as a function of azimuth of the signal, and that the helmet would most seriously impact performance at the $45^{\circ}$ azimuth. The perceptual results were consistent with these analyses. With or without the helmet listeners tended to be more accurate at $0^{\circ}$ azimuth compared to either $45^{\circ}$ or $90^{\circ}$, and the presence of the helmet always degraded perception relative to the no-helmet condition. It is likely that in real world conditions the helmet would impair hearing more seriously than that observed in the present study. In our study we equalized RMS levels so that the signal level was consistent across the hearing conditions (both helmet and azimuth). In the real world, as players orient their head so that the signal is not broadcast from $0^{\circ}$ azimuth, the signal level would be distorted with both positive and negative interference, while crowd noise would be constant producing a more challenging $\mathrm{S} / \mathrm{N}$ ratio. Thus, it is likely that our measurements of the impediment to hearing posed by the helmet are conservative.

Some football players may sense that wearing a helmet improves their hearing, and the present findings conflict with these anecdotal reports. However, in our experiments all subjects listened to signals influenced by the same acoustic geometry posed by the shape of the Neumann artificial head and Adams' helmet. On a football team the head and face morphology of the players differ, and different sizes and brands of helmets, face masks, and chin straps may change the aural experience for some head-helmet configurations. Additional research should address this possibility.

As noted above, the constructive interference at $45^{\circ}$ that was found principally in the speech range. While this interference would be expected to increase the amplitude of the signal, so too would it increase the noise. It may be that because the 12-talker babble was more spectrally uniform that it effectively created a more difficult $\mathrm{S} / \mathrm{N}$ in the speech range relative to other spectral regions. Our prior work in habitat acoustics [1-4] has suggested that under some conditions complex signals (such as vocalizations) may be scattered and reflected in such a way that different frequency components of the signal are propagated along different pathways, and as a results the signal incident at the listener's ears is distorted by an apparent frequency specific phase shift or time delay. Other distortion processes may be at work as well, but it is possible that the acoustics of the helmet may under some azimuths increase the RMS level of the signal, but simultaneously distort the waveform so that speech detection is impaired.

In every experiment the semantic context of the sentence was found to affect performance. Consistent with numerous previous studies, participants performed better when semantic context was high compared to the low-context condition [13, 14]. This finding once again indicates the importance of top-down processing on the disambiguation of speech in difficult listening situations. Of interest here is the observation that the context tended to cause a greater improvement as the listening conditions became easier. For many of the conditions comparing helmet to no-helmet listening, and in most of the comparisons between two listening angles with the helmets, participants tended to make better use of context cues in whichever condition was the easiest (i.e., no-helmet, or better listening angles). The notable exception was found with the comparison contrasting $45^{\circ}$ and $90^{\circ}$ azimuths. In this comparison the largest performance improvement was found for low-context and not high-context sentences.

In summary these data indicate that the helmet can induce changes to a speech signal that can reduce the effectiveness of aural communication. The magnitude of this reduction will be largely dependent on the listening angle for the helmet wearer, and the level of the background noise.

To our knowledge the present study is the first investigation into the perceptual impediment imposed by the design of football helmets. An interesting question is whether minor changes in the design of the helmet could be made to more closely approximate the spectral characteristics of natural (no-helmet) human hearing. Certainly the level of competitiveness in college and professional football suggests that even a minor improvement in on-field communication could provide a game changing advantage. Overall, there have been very few studies that have explored how hearing is affected by wearing any type of helmet [6,7], and the research design of the studies addressing hearing with motorcycle helmets [6], and those examining hearing with military helmets [8-10] are so dissimilar that it is difficult to make meaningful comparisons across these literatures. The engineering of protective head gear would benefit from the development of an auditory assessment protocol that would measure the impact of variations in helmet materials, design and fit on acoustic communication in real-world settings, and the wearers of helmets in a wide variety of industrial, military, and athletic settings would correspondingly benefit.

\section{ACKNOWLEDGEMENTS}

This research was supported in part by a grants provided by Adams USA, and the University of South Alabama. 


\section{REFERENCES}

[1] Brown $\mathrm{CH}$, Gomez R. Functional design features in primate vocal signals: The acoustic habitat and sound distortion. In: Nishida T, McGrew TWC, Marler P, Pickford M, de Waal FMB, Eds. Topics in Primatology, Human Origins. Tokyo: University of Tokyo Press 1992; vol. 1: pp. 177-98.

[2] Brown CH, Waser PM. Environmental influences on the structure of primate vocalizations. In: Todt D, Goedeking P, Symmes D. Eds. Primate Vocal Communication. Berlin: Springer-Verlag 1988; pp. 51-66.

[3] Brown CH, Sinnott JM. Cross species comparisons of vocal perception. In: Greenberg S, Ainsworth WA, Eds. Listening to Speech: An Auditory Perspective. Mahwah, N.J: Lawrence Erlbaum Associates 2006; pp. 183-202.

[4] Waser PM, Brown CH. Habitat acoustics and primate communication. Am J Primatol 1986; 10: 135-54.

[5] Kalikow D, Stevens K, Elliott L. Development of a test of speech intelligibility in noise using test materials with controlled word predictability. J Acoust Soc Am 1997; 61: 1337-51.

[6] Aldman B, Gustaffson H, Nygren A, Wersall J. Hearing and motorcycle helmets. J Traffic Med 1983; 11: 42-4.

[7] McKnight AJ, McKnight AS. The effects of motorcycle helmets upon seeing and hearing. Accident Anal Prevent 1995; 27: 493501.

[8] Scharine AA, Henry PP, Binseel MS. An evaluation of selected communications assemblies and hearing protection systems: a field study conducted for future force warrior integrated headgear integrated process team (Performing Organization Report No. ARL-TR-3475). Aberdeen Proving Ground, MD: U.S. Army Research Laboratory 2005.

[9] Scharine AA, Letowski TR. Factors affecting auditory localization and situational awareness in the urban battlefield (Performing Organization Report No. ARL-TR-3474). Aberdeen Proving Ground, MD: U.S. Army Research Laboratory 2005.

[10] Weatherless RA, Wilson RM, Garrett L, Letowski TR, Binseel MS. Effects of the advanced combat helmet $(\mathrm{ACH})$ and selected communication and hearing protection systems (C\&HPSs) on speech communication: Talk through systems (Performing Organization Report No. ARL-TR-4078). Aberdeen Proving Ground, MD: U.S. Army Research Laboratory 2007.

[11] Scharine A. The impact of helmet design on sound detection and localization. J Acoust Soc Am 2005; 117: 2561.

[12] Bilger RC, Nuetzel JM, Rabinowitz WM, Rzeczkowski C. Standardization of a test of speech perception in noise. J Speech Hear Res 1984; 27: 32-48.

[13] Grant KW, Seitz PF. The recognition of isolated words in sentences: Individual variability in the use of sentence context. J Acoust Soc Am 2000; 107: 1000-1011.

[14] Lewis HD, Benignus VA, Muller KE, Malott CM. Babble and random-noise masking of speech in high and low context cue conditions. J Speech Hear Res 1988; 31: 108-14.

(c) Gordon et al.; Licensee Bentham Open.

This is an open access article licensed under the terms of the Creative Commons Attribution Non-Commercial License (http://creativecommons.org/licenses/by$\mathrm{nc} / 3.0 /$ ) which permits unrestricted, non-commercial use, distribution and reproduction in any medium, provided the work is properly cited. 